\title{
Combination of Two Matrices Results in Improved Performance of MALDI MS for Peptide Mass Mapping and Protein Analysis
}

\author{
Sabrina Laugesen and Peter Roepstorff \\ Department of Biochemistry and Molecular Biology, University of Southern Denmark, Odense, Denmark
}

\begin{abstract}
A new sample preparation method for MALDI based on the use of a mixture of the two commonly used matrices, DHB and CHCA, is described. The matrix mixture preparation results in increased sequence coverage and spot-to-spot reproducibility for peptide mass mapping compared to the use of the single matrix components. This results in more reliable protein identification in proteomics studies and facilitates automated data acquisition. This method shows better tolerance towards salts and impurities, eliminating the need for pre-purification of the samples. It has also been found to be advantageous for the analysis of intact proteins, and especially for glycoproteins. The mixture allows the presence of rather high concentrations of urea in the sample solutions. (J Am Soc Mass Spectrom 2003, 14, 992-1002) (c) 2003 American Society for Mass Spectrometry
\end{abstract}

0 ince its introduction in 1985-1988, matrix-assisted laser desorption/ionization (MALDI) has revolutionized biological mass spectrometry [1]. Karas and Hillenkamp embedded the analytes in a UV-absorbing matrix, first tryptophan [2] and then nicotinic acid [3-5] while Tanaka et al. [6], used glycerol and finely divided metal powder. The technique underwent a fast development over the following years, resulting in the change of the preferred laser wavelength $[7,8]$, the discovery of new matrices [8-14], and sample preparation methods [4, 15-19]. The MALDI process generally involves deposition of a dilute solution of the analyte of interest and a highly concentrated matrix solution onto a target, either separately or together in the form of an admixture of the two solutions and allowing them to dry. The desorption/ionization process has been described as a fast solid-state to gas-phase transition initiated by the absorption of the laser energy, followed by a jet expansion of the gas plume into the vacuum [20-22].

Among the strengths of the matrix-assisted laser desorption/ionization (MALDI) technique are the high sensitivity, the ability to analyze complex mixtures of biomolecules (e.g., proteolytic protein digests) [8, 11], its ability to tolerate relatively high concentrations of contaminants (such as buffers and salts) [22-27] and the possibility for high sample throughput. These features are the reason why MALDI-time-of-flight mass spectro-

Published online July 24, 2003

Address reprint requests to Dr. P. Roepstorff, Department of Biochemistry and Molecular Biology, Campusvej 55, Odense DK-5230, Denmark. E-mail: roe@bmb.sdu.dk metry (TOF-MS) is considered to be one of the most powerful techniques in proteomic studies.

Despite increased knowledge of the MALDI process, matrix selection and optimization of the preparation protocol are still empirical procedures. Numerous matrix candidates have been evaluated. Some benzoic acid and cinnamic acid derivatives have been found to be good MALDI matrices. In particular, 2,5-dihydroxybenzoic acid (DHB) [12] and $\alpha$-cyano-4-hydroxycinnamic acid (CHCA) [13] have become favored matrices for the analysis of peptides and small proteins. Selection of an appropriate sample preparation method for the analyte is a critical point in order to obtain high quality MALDI mass spectra. Even though tremendous efforts have been made in the development of new protocols, the dried-droplet method described originally by Karas et al. 1988 [4], is still currently used in many laboratories because it is simple, effective, and can tolerate moderate concentrations of contaminants.

A number of approaches have been made to overcome the matrix selectivity problem, either by using additives to matrix preparations [28, 29] or by mixtures of matrix compounds (e.g., super DHB) [30-32]. Here we demonstrate that the combined use of two common matrices (CHCA and DHB) mixed in the dried-droplet preparation results in increased surface homogeneity and spot-to-spot reproducibility, compared to preparations with a single matrix compound. In addition, increased signal-to-noise ratio improved sequence coverage for peptide mapping, and increased tolerance to impurities (e.g., salts and urea) is obtained. The matrix mixture preparation also results in high quality spectra of proteins in the mass range $\sim 10-80 \mathrm{kDa}$. 


\section{Experimental}

\section{Chemicals and Standards}

Matrices. The following matrices were used: $\alpha$-cyano4-hydroxycinnamic acid (CHCA), 2,5-dihydroxybenzoic acid (DHB), and sinapinic acid (SA) obtained from Aldrich (Steinheim, Germany).

Standard peptides and proteins. Bovine serum albumin (BSA), alcohol dehydrogenase (ADH), lactoglobulin and ribonuclease B, were from Sigma (Deisenhofen, Germany). MALDI Quality Standard peptides (oxytocin Mw 1007.20, arginine-8-vasopresin Mw 1084.25, angiotensin I Mw 1281.49, somatostatin Mw 1637.90, chicken atrial natriuretic peptide Mw 3160.66, human r insulin Mw 5807.70 and $\mathrm{r}$ hirudin Mw 6963.52) were from Hewlett-Packard (Palo Alto, CA). The peptides and proteins were prepared freshly in 5\% FA prior to use. All chemicals were used as obtained without further purification.

Matrix solutions. The following matrix solutions were prepared: CHCA: $20 \mu \mathrm{g} / \mu \mathrm{L}$ in $\mathrm{ACN}, 5 \%$ formic acid (FA) (70:30, vol/vol). DHB: $20 \mu \mathrm{g} / \mu \mathrm{L}$ in $\mathrm{ACN}, 0.1 \%$ trifluoroacetic acid (TFA) (70:30, vol/vol). SA: 20 $\mu \mathrm{g} / \mu \mathrm{L}$ in $\mathrm{ACN}, 0.1 \%$ TFA (70:30, vol/vol).

All solvents used were sequence grade from Sigma (Deisenhofen, Germany). The water was purified on a Milli-Q system (Millipore, Bedford, MA).

\section{In-Gel Digestion}

Spots were cut out from 2-D silver stained (barley grain proteins) or 1-D Coomassie-stained (standard proteins: BSA, ADH, and lactoglobulin) electrophoresis gels and subjected to in-gel trypsin digestion according to Shevchenko et al. [33], with minor modifications. The gel pieces were swollen in a digestion buffer containing $50 \mathrm{mM} \mathrm{NH}_{4} \mathrm{HCO}_{3}$ and $12.5 \mathrm{ng} / \mu \mathrm{L}$ of trypsin (modified porcine trypsin, sequencing grade, Promega, Madison, $\mathrm{WI}$ ) in an ice bath. After $30 \mathrm{~min}$ the supernatant was removed and discarded, $20 \mu \mathrm{L}$ of $50 \mathrm{mM} \mathrm{NH} \mathrm{NHCO}_{3}$ were added to the gel piece and the digestion allowed to proceed at $37^{\circ} \mathrm{C}$ overnight. The supernatant containing tryptic peptides was dried by vacuum centrifugation. Prior to MALDI analysis, the peptide mixture was redissolved in $5 \mu \mathrm{L}$ of $5 \% \mathrm{FA}$.

\section{Sample Preparation for MALDI MS}

Three different sample preparation methods were used: (a) The dried-droplet method [4]: $0.5 \mu \mathrm{L}$ of analyte solution was mixed with $0.5 \mu \mathrm{L}$ of matrix solution on a plastic sheet (a standard transparency sheet) and then applied to the target and allowed to dry. Mixing was also tried directly on the target, but premixing on a plastic sheet resulted in slightly better spectra. Therefore all results described have been prepared by pre- mixing on a plastic sheet. (b) The matrix mixture: The mixture was prepared by making separate solutions of the two matrices, each in its specific solvent. The solutions were then combined in a 1:1 volume ratio of the CHCA and DHB solutions. The resulting mixture was vortexed for a few seconds and used as the matrix solution in a dried-droplet preparation as described above. (c) The nano-column purification of in-gel digests: The in-gel digests were purified as described [18] with some modifications. A column consisting of 100300 nL of POROS R2 material (PerSeptive Biosystems, Framingham, MA) was packed in a constricted GELoader tip (Eppendorf, Hamburg, Germany). A 1.25 mL syringe was used to force liquid through the column by applying a gentle air pressure. The column was equilibrated with $20 \mu \mathrm{L}$ of $5 \% \mathrm{FA}$ and the analyte solution was added. The column was washed with $20 \mu \mathrm{L}$ of $5 \%$ FA and the bound peptides subsequently eluted directly onto the MALDI target with $0.5 \mu \mathrm{L} \mathrm{CHCA}$ solution $(20 \mu \mathrm{g} / \mu \mathrm{L}$ in $\mathrm{ACN}, 5 \% \mathrm{FA}, 70: 30$, vol/vol).

\section{Mass Spectrometric Analysis}

Peptide mass mapping was performed on a Bruker REFLEX MALDI-TOF mass spectrometer upgraded to a reflex IV (Bruker-Daltonics, Bremen, Germany). Positively charged ions were analyzed in the reflector mode, using delayed extraction. The spectra were obtained by randomly scanning the sample surface when using $\mathrm{CHCA}$ and the matrix mixture preparations. For DHB, the spectra were obtained by searching for the "sweet" spots (needles) throughout the entire sample surface. Typically, 100 shots were averaged to improve the signal-to-noise ratio. All spectra were analyzed using the $m / z$ software (Proteometrics, New York). All spectra depicted are original data without any processing such as filtering or background substraction. The laser fluence was adjusted to the threshold for each matrix used. Spectra were calibrated using trypsin autolysis products $(\mathrm{m} / \mathrm{z} 842.509$ and 2211.104) for two points internal calibration [34] resulting in a mass accuracy of $<50$ ppm. Protein identification was performed by searching in a non-redundant protein sequence database (NCBI) using the Mascot program (http:/ / www.matrixscience. com). The following parameters were used for database searches: Monoisotopic mass accuracy <50 ppm, missed cleavages 1, allowed modifications carbamidomethylation of cysteine (complete) and oxidation of methionine and pyroglutamic acid (partial). The peptide mass maps and the protein identification were evaluated as described by Jensen et al. 1998 [35].

MALDI spectra of proteins were acquired on a Voyager-DE STR BioSpectrometry Workstation instrument (Applied Biosystems, Foster City, CA). Positively charged ions were analyzed in the linear mode, using delayed extraction. The sample spots were analyzed as described above. The instrument was calibrated using appropriate proteins that have masses flanking the known mass of the protein of interest as external 


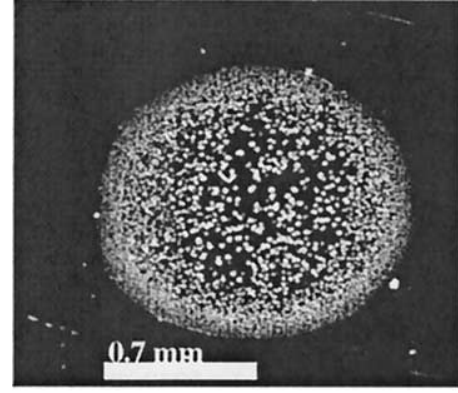

(a) $\mathrm{CHCA}$

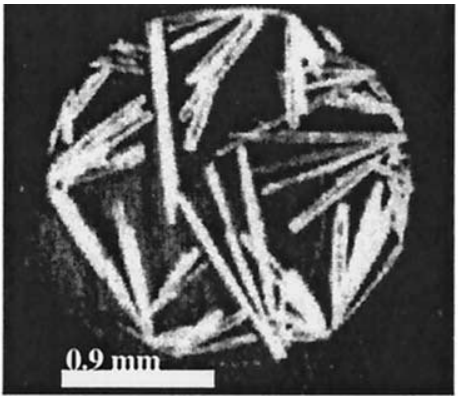

(b) DHB

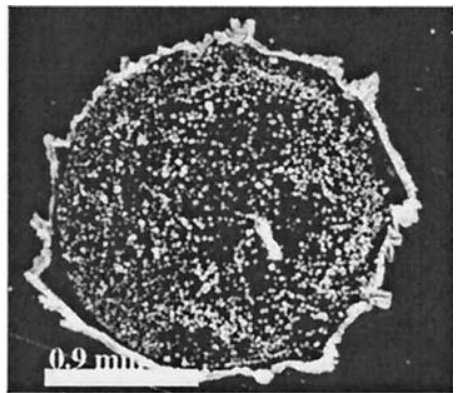

(c) Matrix mix

Figure 1. Microphotographs obtained with (a) CHCA, (b) DHB, and (c) matrix mixture preparations in the dried-droplet method. A $0.5 \mu \mathrm{L}$ volume of acidified analyte was mixed on a plastic sheet with $0.5 \mu \mathrm{L}$ volume of matrix solution. The mixture was then transferred onto the target and left to dry.

standards, such that a mass accuracy of at least $0.1 \%$ was achieved for each standard. All the spectra were analyzed using Data Explorer software version 4.0 (Applied Biosystems, Foster City, CA).

\section{Results}

\section{Sample Preparation Morphology and Homogeneity}

A number of different matrix solutions were tested. It was found that the acid used (FA or TFA) influences the quality of the spectrum, confirming previously published observations [36]. In our experience the best reproducibility, signal-to-noise ratio, and reduced ion suppression are obtained when CHCA is prepared with FA and DHB with TFA. Also for the matrix mixture preparation, where the two matrix solutions are mixed, the respective solvents give the best results.

Figure 1 shows microphotographs of sample preparations using $\mathrm{CHCA}, \mathrm{DHB}$, and the matrix mixture prepared by the dried-droplet method. Inspection of the sample structure under microscope showed that CHCA resulted in formation of small crystals that are unevenly distributed over the sample surface, i.e., areas with lower crystal densities at the center of the preparation (Figure 1a). DHB formed large crystal needles pointing inward from the rim (Figure 1b). Combination of the two matrices showed better crystal distribution over the sample preparation, with DHB-like crystals forming short needles at the rim and evenly distributed CHCAlike crystals inside the rim (Figure 1c). Changing the volume ratio in favour of one matrix (e.g., CHCA:DHB in 2:1) resulted in a shift of the crystal type and distribution towards that of the dominant matrix.

The surface heterogeneity observed for CHCA did not prevent formation of analyte ions from the entire surface. However, the ion signal intensity was found to vary strongly depending on the position. The DHB preparation required search for "sweet" spots in the rim. Spectrum acquisition is therefore time consuming and difficult to reproduce, in general agreement with previous observations $[12,19,29]$. For the matrix mixture preparation, good quality spectra with intense signals were in most cases obtained from the entire preparation surface including the rim. Furthermore, the crystal appearance was unchanged after several shots at the same position. In order to investigate the nature of the crystal structures in the matrix mixture preparation, we acquired MALDI spectra from different parts of the surface and examined $\mathrm{m} / \mathrm{z}$ range $0-500$ for the presence of matrix-related ions. The laser fluence required to obtain ions from the rim was twice that needed to obtain ions from the centre of the preparation. The rim seemed to be DHB crystals, based on the observation of DHB related ions and absence of CHCA related ions. The central part yielded CHCA related ions. However, the presence of DHB crystals in the center cannot be excluded since the energy used for acquiring data from this region was half what is needed to ionize DHB. Increasing the laser fluence resulted in an increase in chemical noise that did not allow observation of DHB related ions.

\section{Sequence Coverage, Signal-to-Noise Ratio, and Resolution}

Three in-gel tryptic digests of standard proteins (BSA, $\mathrm{ADH}$, and lactoglobulin) were used to compare the matrix mixture, CHCA, and DHB preparations. Each protein was loaded on 1-D gel, Coomassie-stained and in-gel digested with trypsin. $0.5 \mu \mathrm{L}$ of digested protein corresponding to approximately $150 \mathrm{fmol}$ was mixed with $0.5 \mu \mathrm{L}$ matrix solution, applied onto the MALDI target, and allowed to dry.

For all three proteins, more peptides are observed with the matrix mixture than with CHCA and DHB preparations, resulting in slightly higher sequence coverage (data not shown). In Figure 2, the results obtained with the tryptic digest of BSA for the three matrix preparations are compared. CHCA (Figure 2a) produced a high level of chemical noise, especially in the low mass region, while DHB (Figure $2 \mathrm{~b}$ ) resulted in a spectrum with very few matrix peaks and better signalto-noise ratio. However, with this matrix it was necessary to search for "sweet" spots (needles). With the 


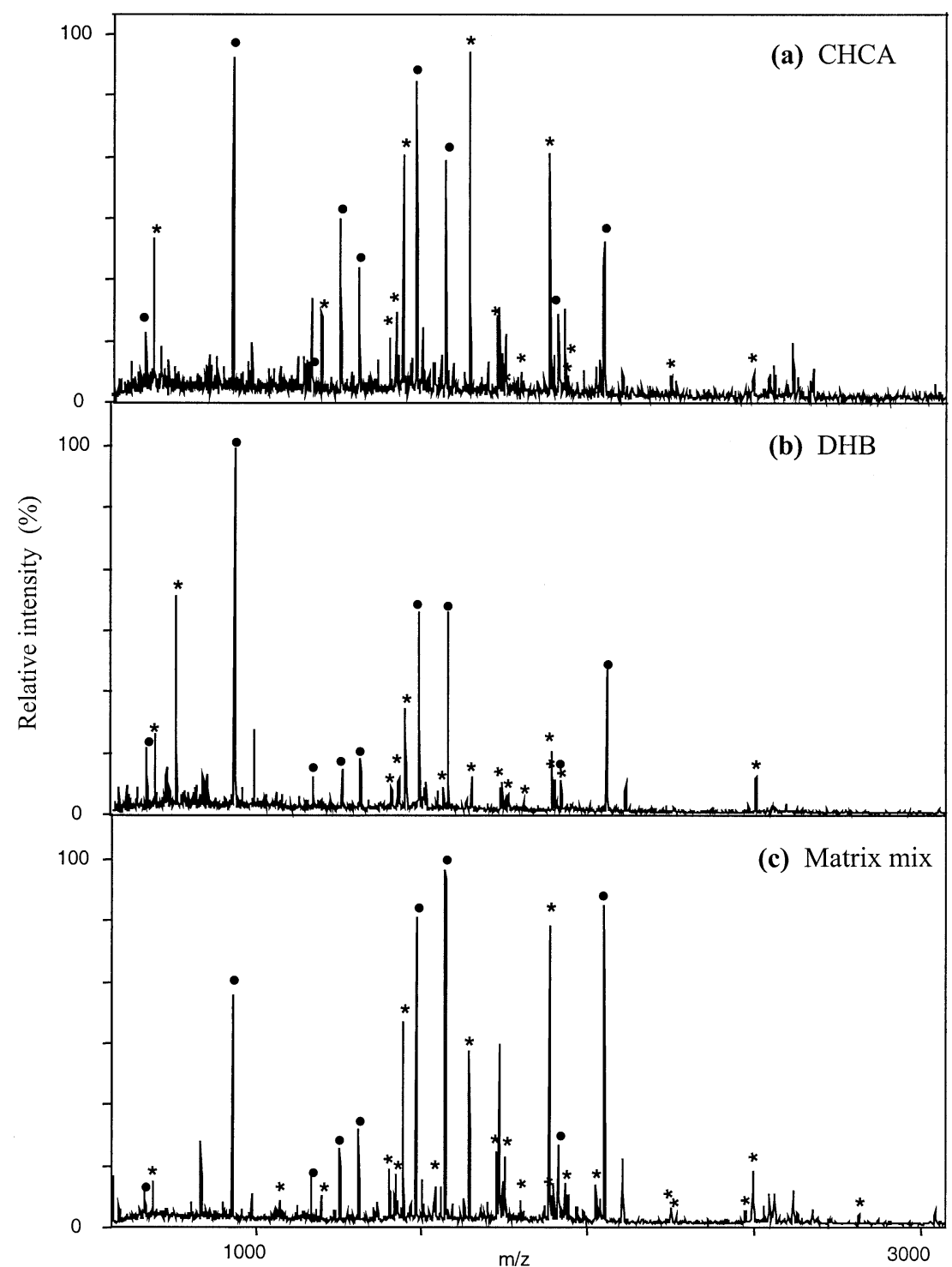

Figure 2. MALDI-TOF mass spectra of a BSA tryptic digest. $0.5 \mu \mathrm{L}$ corresponding to approximately $150 \mathrm{fmol}$ of the digest was mixed with $0.5 \mu \mathrm{L}$ matrix solution, and applied onto the target in the dried-droplet method. The spectra were obtained with the preparations: (a) CHCA, (b) DHB, and (c) matrix mixture. Stars and circles indicate signals matching tryptic peptides of BSA. Circles indicate peaks used for the signal-to-noise ratio measurements given in Table 1.

matrix mixture (Figure 2c), a pronounced suppression of matrix ions was observed in the low mass region compared to CHCA. In addition, most of the peaks showed improved signal-to-noise ratio, compared to CHCA and DHB. Table 1 summarizes peak signal-tonoise ratio obtained for the BSA, indicated with a circle in Figure 2. All the peaks above $\mathrm{m} / \mathrm{z} 1000$ show 2- to 3 -fold increase in signal-to-noise ratio with the matrix mixture preparation compared to the single matrix components. DHB resulted in better signal-to-noise ratio below $\mathrm{m} / \mathrm{z} 1000$ due to reduced chemical noise and matrix signals in this region. The matrix mixture and CHCA showed better resolution than DHB for the larger peptides, e.g., 9200 at $\mathrm{m} / \mathrm{z} 1907.9$ compared to 5600 for DHB. At $\mathrm{m} / \mathrm{z}$ below approximately 1500 no clear tendency could be observed (data not shown).
Table 1. Signal-to-noise ratio is compared for the different matrix preparations (CHCA, DHB, and matrix mixture)

\begin{tabular}{lccc}
\hline \multirow{2}{*}{$\begin{array}{l}\text { Observed } \\
\text { peptides }\left(\mathrm{MH}^{+}\right)^{\mathrm{a}}\end{array}$} & \multicolumn{3}{c}{ Signal-to-noise ratio } \\
\cline { 2 - 4 } & CHCA & DHB & Matrix mix $^{\mathrm{b}}$ \\
\hline \hline 689.4 & 5 & 6 & 4 \\
927.5 & 13 & 37 & 23 \\
1163.6 & 5 & 4 & 7 \\
1250.6 & 8 & 5 & 13 \\
1305.7 & 7 & 6 & 14 \\
1479.8 & 16 & 19 & 38 \\
1567.7 & 12 & 28 & 51 \\
1907.9 & 5 & 5 & 7 \\
2045.0 & 10 & 26 & 61
\end{tabular}

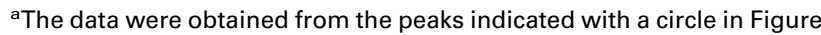
2. ${ }^{\text {bS }}$ Signal-to-noise ratio was calculated using $\mathrm{m} / \mathrm{z}$ software (Proteometrics, New York, USA). 
Table 2. Comparison of sequence coverage obtained from $0.5 \mu \mathrm{L}$ of digestion supernatant from a low level silver-stained 2D gel using the three preparations (CHCA, DHB and matrix mixture) in the dried-droplet method and the nano-column method using CHCA

\begin{tabular}{|c|c|c|c|c|c|}
\hline \multirow[b]{2}{*}{ Spot No. } & \multicolumn{3}{|c|}{ Dried droplet method } & \multirow{2}{*}{$\frac{\text { Nano-column }}{\text { CHCA }}$} & \multirow[b]{2}{*}{ Protein ID } \\
\hline & $\mathrm{CHCA}$ & DHB & Matrix mix & & \\
\hline 1 & $35 \%$ & $35 \%$ & $35 \%$ & $35 \%$ & $\alpha$-amylase P04063 \\
\hline 2 & $28 \%$ & $28 \%$ & $28 \%$ & $28 \%$ & $\alpha$-amylase J04202 \\
\hline 3 & $24 \%$ & $24 \%$ & $24 \%$ & $24 \%$ & $\alpha$ amylase P04063 \\
\hline 4 & $17 \%$ & $28 \%$ & $37 \%$ & $24 \%$ & Serpin X97636 \\
\hline 5 & $12 \%$ & $15 \%$ & $21 \%$ & $19 \%$ & Protein Z X97636 \\
\hline 6 & $60 \%$ & $71 \%$ & $74 \%$ & $61 \%$ & Protein Z X97636 \\
\hline 7 & $15 \%$ & $18 \%$ & $18 \%$ & $15 \%$ & Beta-glucosidase P26204 \\
\hline 8 & $60 \%$ & $59 \%$ & $60 \%$ & $21 \%$ & Ribulose phosphate carboxylase Q9TK10 \\
\hline 9 & $20 \%$ & $21 \%$ & $23 \%$ & $20 \%$ & $\beta$-amylase AF300800 \\
\hline 10 & $20 \%$ & $20 \%$ & $21 \%$ & $20 \%$ & $\beta$-amylase AF300800 \\
\hline
\end{tabular}

\section{Application to Proteomic Studies}

In order to investigate the performance of the new preparation in proteomic studies, CHCA, DHB, and the matrix mixture were compared using dried-droplet preparation for analysis of in-gel digests from a study of the barley grain proteome. Ten spots from low-level silver stained spots were excised from a 2-D gel, in gel digested and $10 \%$ of each tryptic digest was used for MALDI analysis. Table 2 summarizes the sequence coverage obtained for each protein using the different matrix preparations. The matrix mixture preparation for all spots showed comparable or better sequence coverage than CHCA and DHB preparations. Figure 3 shows the spectra obtained from the digest of the protein in spot 6 (Protein $\mathrm{Z}$ ) using the three preparations. All spectra are of reasonable quality. Differences are observed for some of the low abundance peaks. One peak at $\mathrm{m} / \mathrm{z} 2238.1$ is missing in the spectrum using CHCA, and another at $\mathrm{m} / \mathrm{z} 591.4$ is missing when using DHB compared to the matrix mixture preparation (insets in Figure 3). The signal-to-noise ratio was comparable for DHB and the matrix mixture preparations, and poor for the CHCA preparation. However, it must be borne in mind that time-consuming search for "sweet" spots was needed to obtain the spectra with DHB. The peak pattern abundances were different for the three preparations, the matrix mixture being closer to $\mathrm{CHCA}$ than DHB preparations.

In order to test the tolerance of the matrix mixture preparation to impurities it was compared to our standard preparation method, which includes a desalting, and concentration step on a nano-column, followed by elution with CHCA matrix solution. Equal aliquots of the digestion supernatant from each spot were loaded directly onto the MALDI target or desalted and concentrated on R2 GELoader tip nano-column. The sequence coverage obtained from each spot from the silverstained 2-D gel is shown in Table 2. In 7 out of 10 analyzed proteins, the matrix mixture preparation resulted in slightly or significantly higher (up to $40 \%$ ) sequence coverage. The signal-to-noise ratio was slightly better after purification on the nano-column
(Figure 4) but considerably fewer peaks were observed. Examination of spectra from all the spots shows that these include signals corresponding to small hydrophilic and large hydrophobic peptides, which are either not retained on the column or not eluted with the matrix solution, respectively. However, for very faint spots in the gel, up-concentration of the in-gel digests on nano-columns was found to be needed to obtain protein identification.

\section{Applications to Intact Proteins}

The matrix mixture was tested to examine its potential for analysis of intact proteins. The results obtained by analysis of ribonuclease $B$, using the different preparation methods, are shown in Figure 5. Approximately 50 fmol of protein was premixed with the matrix solution and deposited onto the MALDI target. 100 shots randomly chosen over the entire sample surface were accumulated with the matrix mixture and CHCA. With DHB, it was necessary to search for "sweet" spots to obtain a spectrum of reasonable quality. Ribonuclease B is a glycoprotein having "N-linked" glycan with the heterogeneous structure $\mathrm{GlCNAc}_{2} \mathrm{Man}_{5-9}$ attached to asparagine $_{60}$ [37]. All spectra show the five glycosylated forms of ribonuclease B corresponding to $\mathrm{GlCNac}_{2} \mathrm{Man}_{5}$ to $\mathrm{GlNNac}_{2} \mathrm{Man}_{9}$, with the mass difference between adjacent ions corresponding to the addition of a mannose unit $(162 \mathrm{Da})$. The resolution of the different glycoforms was best with the matrix mixture preparation. The spectrum obtained with CHCA showed poor resolution while those obtained with DHB showed an extensive adducting resulting in a high mass tailing. The performance of the matrix mixture compared to the other matrices was also tested with a number of standard non-glycosylated proteins (e.g., insulin, myoglobin, trypsin, BSA, etc). Similar or slightly improved spectra were obtained with the matrix mixture preparation. However the effect was not as pronounced as with ribonuclease $B$, which might indicate that the new matrix preparation is favorable for analysis of glycoproteins. To support this, the matrix mixture was tested 


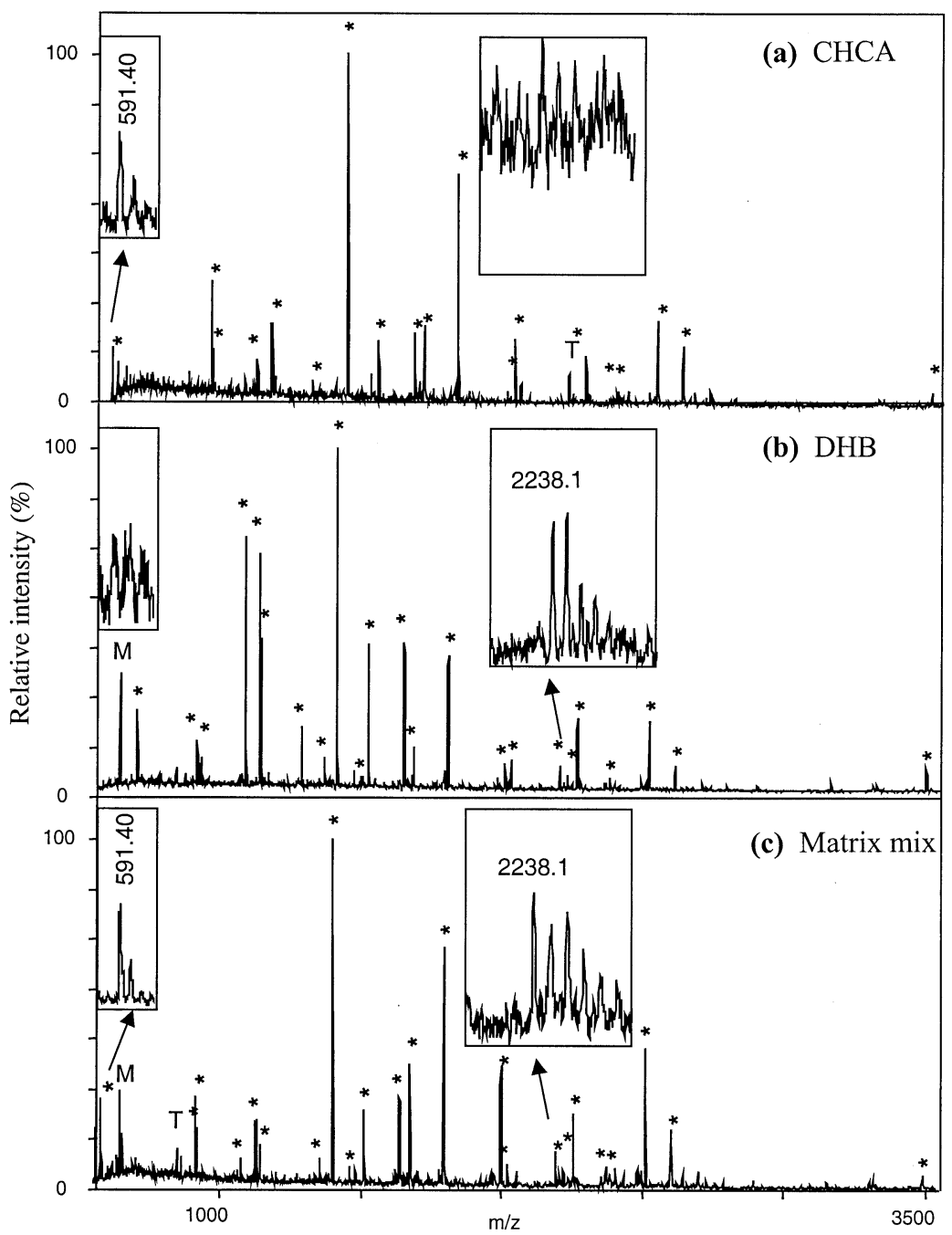

Figure 3. MALDI-MS spectra obtained from $0.5 \mu \mathrm{L}$ of supernatant digest from low-level silverstained 2-D gel spot 6 using the dried-droplet method. The spectra were obtained with the preparations (a) CHCA, (b) DHB, and (c) matrix mixture. Stars indicate signals matching protein z-type serpin from barley. Commonly observed matrix and trypsine auto-digest signals are indicated by $\mathrm{M}$ and $\mathrm{T}$ respectively. The insets show peaks missing in some of the spectra.

for analysis of a number of recombinant glycoproteins. Figure 6 shows the spectrum obtained with a recombinant variant of glucoamylase ( $\sim 71 \mathrm{kDa})$ from Aspergillus niger expressed in Pichia pastoris. The protein was purified by affinity chromatography followed by an ion exchange chromatography. Five hundred fmol of the protein was applied onto the target using the three different matrices. An intense signal was obtained with the matrix mixture, whereas signals close to the noise level were obtained with CHCA and DHB preparations. Comparable improvements were obtained with matrix mixture preparation for all tested glycoproteins. In one case, this preparation was the only one that resulted in a spectrum of the protein.

It was suspected that the reason for failure with DHB and CHCA was a rather high content of contaminants (possibly urea) in the samples. The matrix preparations were therefore tested for their tolerance to the presence of urea. Inspection of the matrix crystals under micro- scope showed poor crystal formation for all preparations when urea was present. However, spectra could still be obtained. Figure 7 shows the spectra obtained with $300 \mathrm{fmol}$ ribonuclease $B$ in the presence of $80 \mathrm{mM}$ urea for all three preparations. Only the spectrum obtained with the matrix mixture shows the resolution of the different carbohydrate heterogeneity. Using $\mathrm{DHB}$, it was not possible to obtain signals at $300 \mathrm{fmol}$ level, and only a broad peak was obtained at $600 \mathrm{fmol}$ (Figure $7 \mathrm{~b}$ ). The matrix mixture preparation could tolerate up to $8 \mathrm{M}$ urea if the protein amount was increased to $6 \mathrm{pmol}$ (data not shown).

\section{Prompt and Metastable Fragmentation}

The three different matrix preparations were also compared for their tendency to induce fragmentation. Methionine oxidation is a common modification easily observed in MALDI spectra. Depending on the matrix 


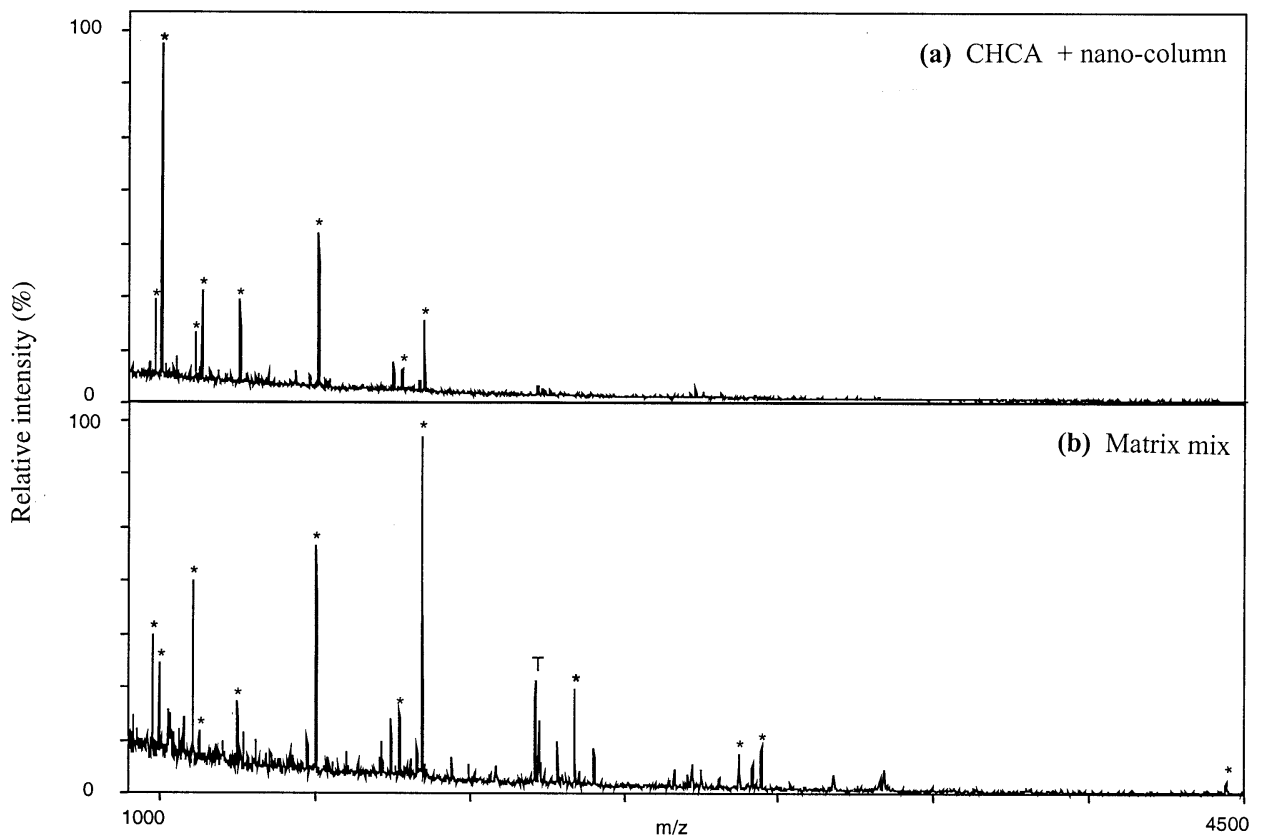

Figure 4. MALDI-MS spectra obtained from $0.5 \mu \mathrm{L}$ of supernatant digest from low-level silver-stained 2-D gel spot 8. The spectra were obtained with (a) nano-column sample preparation using CHCA as matrix, (b) matrix mixture preparation using the dried-droplet method. Stars indicate signals matching ribulose phosphate carboxylase. Signals from trypsin auto-digest peptides are indicated by $\mathrm{T}$.

conditions, prompt and metastable side chain fragmentation are observed. Figure 8 shows the region of the spectrum of a tryptic digest of lactoglobulin containing the peaks derived from the methionine containing peptide $\left(\mathrm{Ala}_{131}-\mathrm{Arg}_{137}\right) \mathrm{Mw}$ 837.06. The non-oxidized and oxidized forms of the peptide are observed at $m / z 837.49$

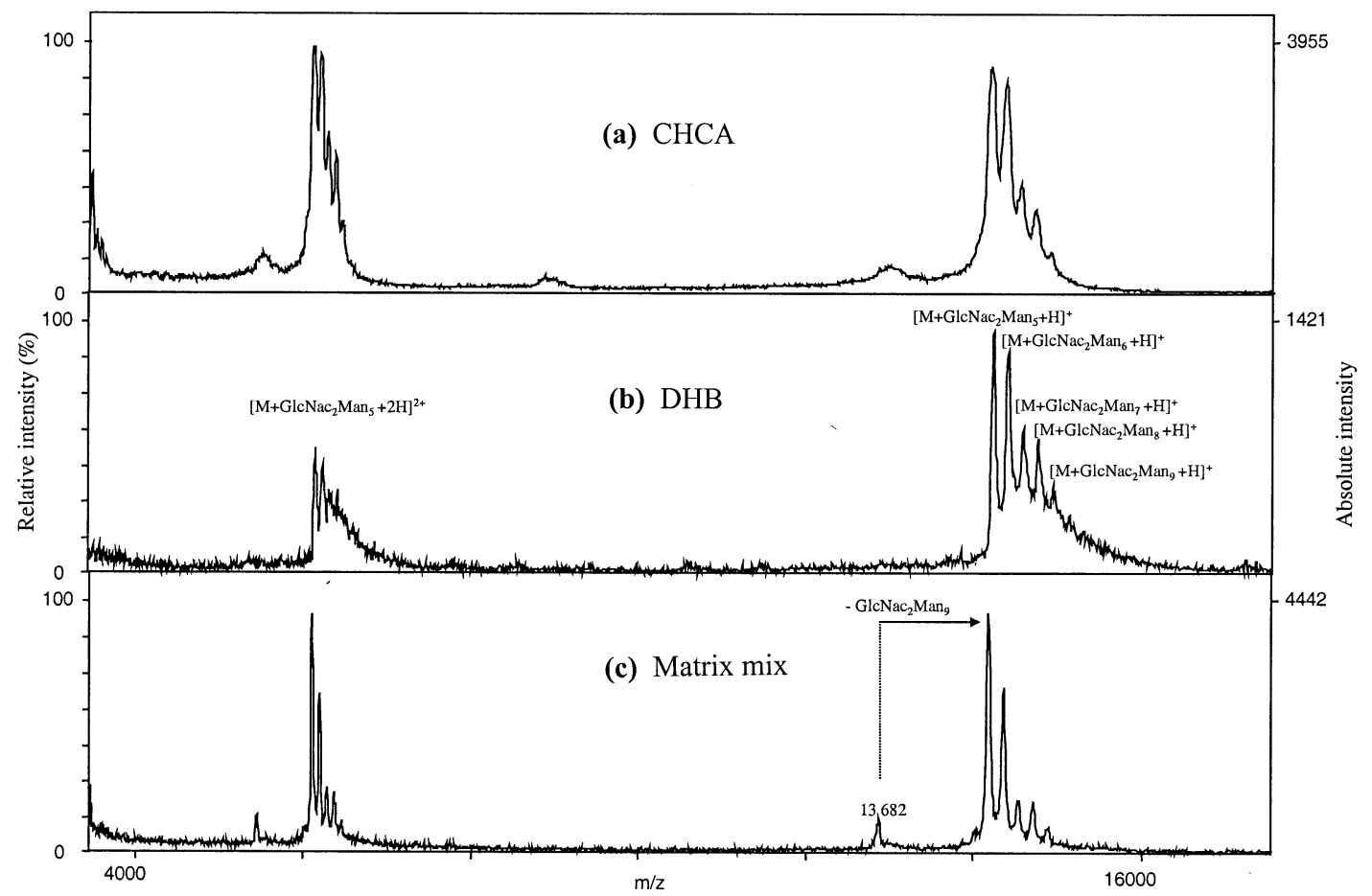

Figure 5. MALDI/Linear-TOF spectra of ribonuclease B. $0.5 \mu \mathrm{L}$ analyte solution corresponding to approximately $50 \mathrm{fmol}$ of the protein was mixed with $0.5 \mu \mathrm{L}$ matrix solution, and applied onto the target with the dried-droplet method. The spectra were obtained with the preparations: (a) CHCA, (b) DHB, and (c) matrix mixture. GlcNac $\mathrm{Man}_{5}$, GlcNac$_{2} \mathrm{Man}_{6}$, GlcNac$_{2} \mathrm{Man}_{7}, \mathrm{GlcNac}_{2} \mathrm{Man}_{8}$, andGlcNac ${ }_{2} \mathrm{Man}_{9}$ correspond to the 5 glycosylated forms of ribonuclease $\mathrm{B}$. 


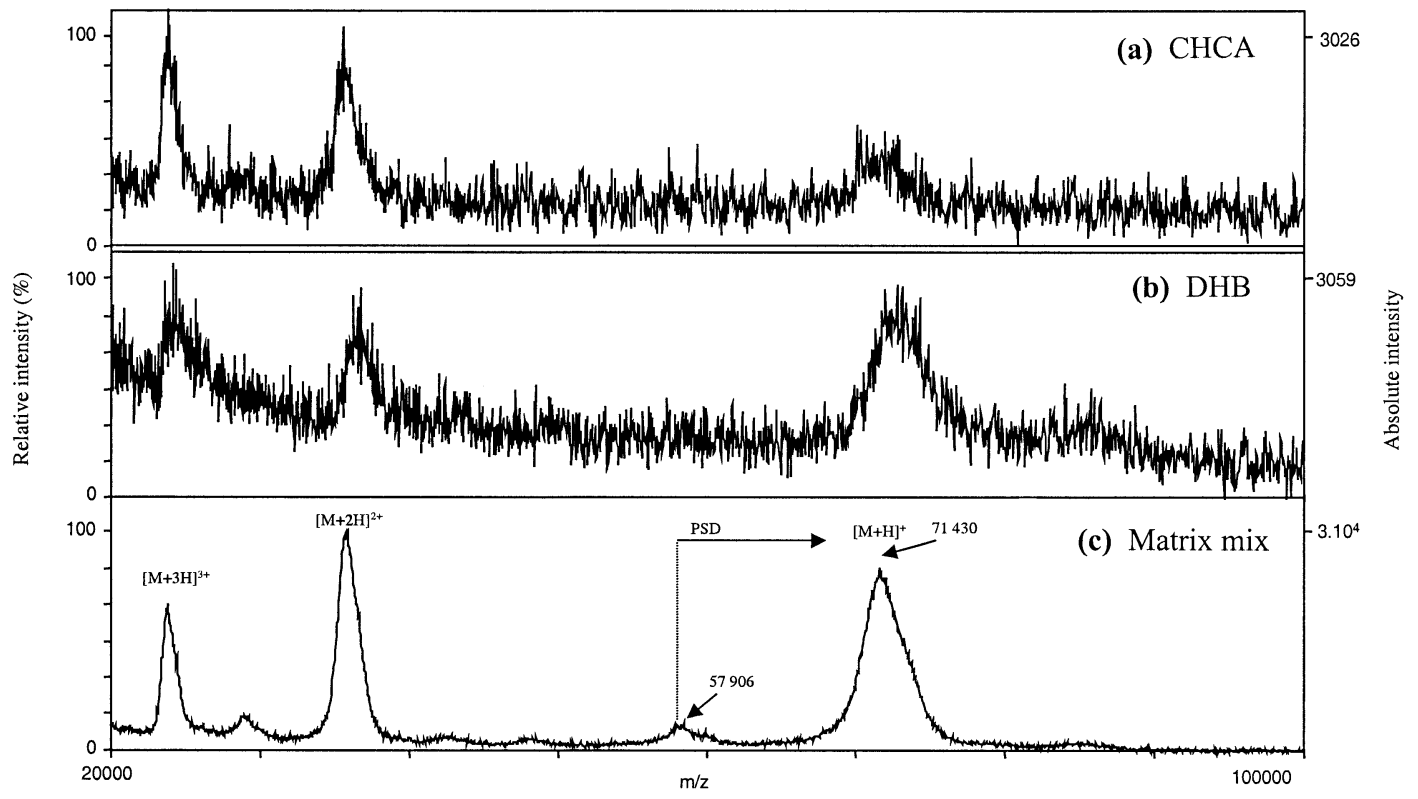

Figure 6. MALDI/Linear-TOF spectra of a recombinant variant of glucoamylase ( $\sim 71 \mathrm{kDa})$ from Aspergillus niger expressed in Pichia pastoris. $0.5 \mu \mathrm{L}$ analyte solution corresponding to approximately $500 \mathrm{fmol}$ of the protein was mixed with $0.5 \mu \mathrm{L}$ matrix solution, and applied onto the target in the dried-droplet method. The spectra were obtained with the preparations: (a) CHCA, (b) DHB, and (c) matrix mixture.

and 853.48 , respectively. The oxidized methionine side chain fragments by loss of methanesulfenic acid, resulting in a peak at $\mathrm{m} / \mathrm{z} 789.51$ due to in-source decay (ISD) and a broad peak at $m / z 796.52$ due to post-source decay (PSD). The position of the latter peak depends on the specific parameters of the MALDI-TOF instrument. ISD

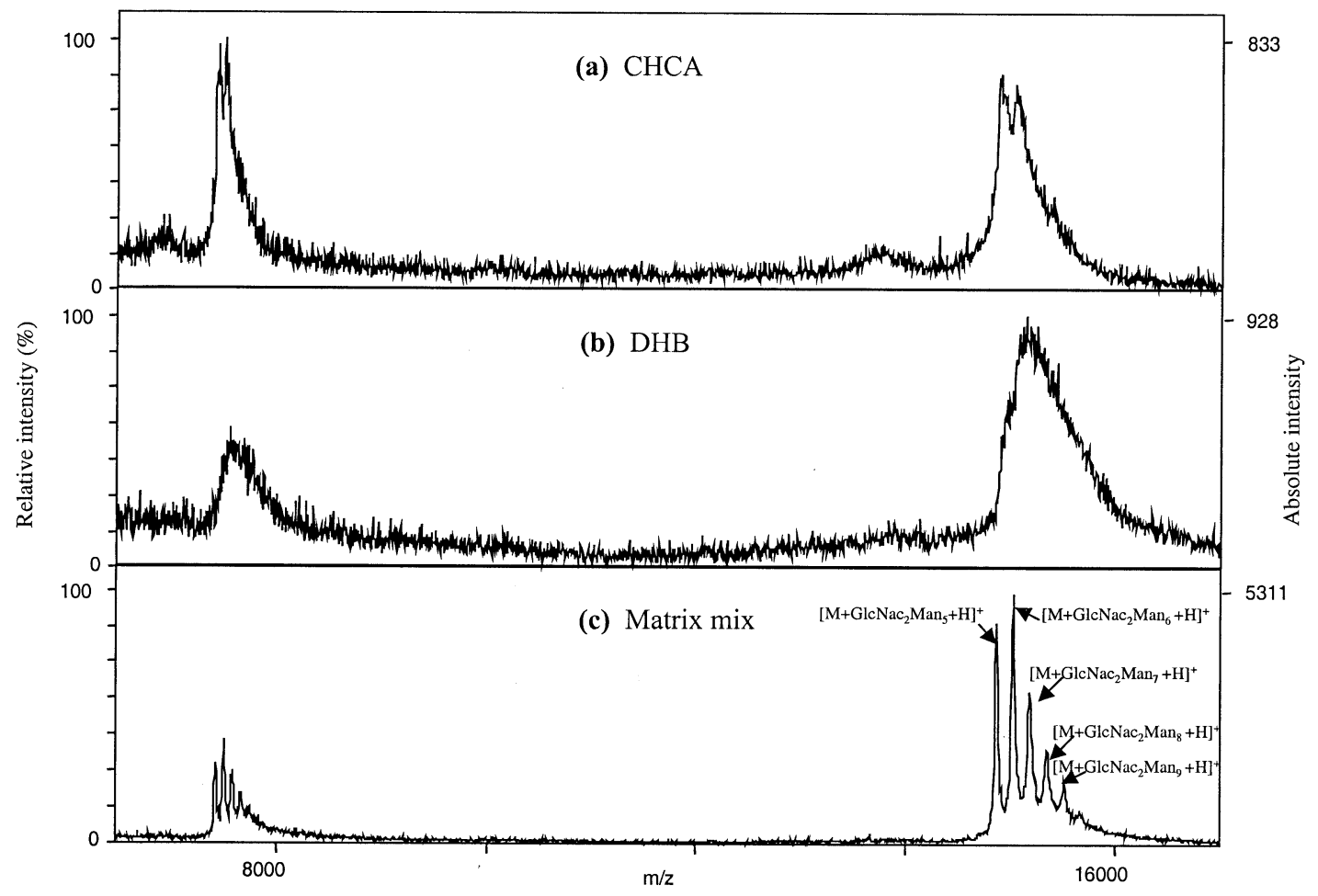

Figure 7. MALDI/Linear-TOF spectra of ribonuclease B in $80 \mathrm{mM}$ urea. $0.5 \mu \mathrm{L}$ of the protein solution was mixed with $0.5 \mu \mathrm{L}$ matrix solution, and applied onto the target in the dried-droplet method. The spectra were obtained with the preparations: (a) $300 \mathrm{fmol}$ of protein using CHCA, (b) $600 \mathrm{fmol}$ using $\mathrm{DHB}$, and (c) $300 \mathrm{fmol}$ using matrix mixture. 


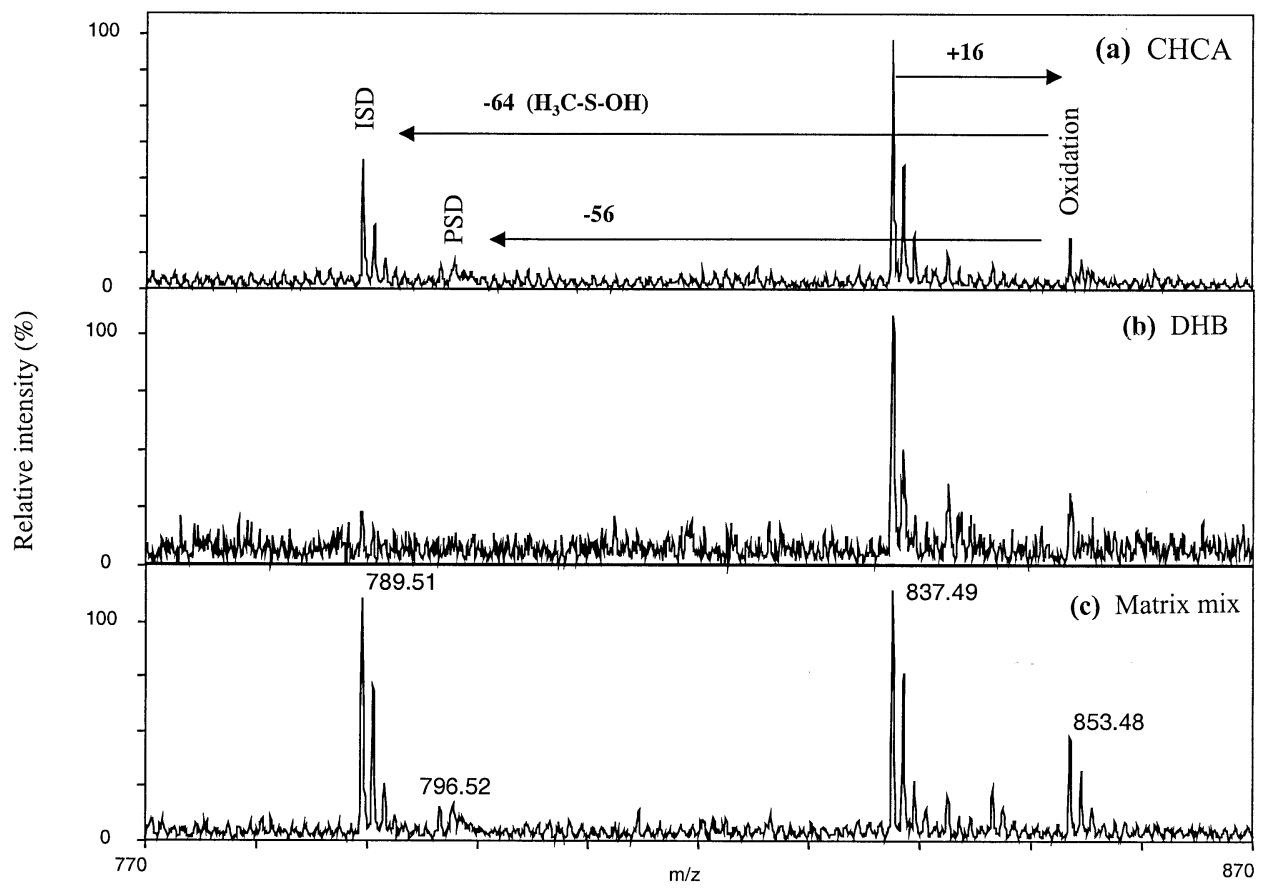

Figure 8. Region of the MALDI-TOF mass spectra of a tryptic digest of lactoglobulin showing the peaks derived from the methionine-containing peptide (Ala131-Arg137). Mw 837.06. 0.5 $\mu \mathrm{L}$ corresponding to approximately $150 \mathrm{fmol}$ of the digest was mixed with $0.5 \mu \mathrm{L}$ matrix solution, and applied onto the target in the dried-droplet method. The spectra were obtained with the preparations: (a) CHCA, (b) DHB, and (c) matrix mixture. The non-oxidized and oxidized forms of the peptide are observed at $\mathrm{m} / \mathrm{z} 837.49$ and $\mathrm{m} / \mathrm{z} 853.48$ respectively. The peaks at $\mathrm{m} / \mathrm{z} 789.51$ and $\mathrm{m} / \mathrm{z} 797.69$ result from in source decay (ISD) and post source decay (PSD) respectively, of the oxidized methionine by side chain fragmentation.

was more prominent when using the matrix mixture than with CHCA preparation. With DHB no fragmentation was observed.

The spectra of ribonuclease B (Figure 5) show a similar tendency. With the matrix mixture preparation, a narrow peak at $m / z 13682$ is observed, most likely caused by the loss of the entire glycan by in-source decay. With CHCA preparation, a rather broad peak caused by loss of the glycan due to a combination of prompt and metastable decay is observed. In addition, the loss of resolution of the different glycans observed for this matrix might be caused by metastable decay of the glycans. With DHB as matrix no fragmentation is observed.

\section{Sensitivity}

The sensitivity of the matrix mixture preparation was compared to DHB and CHCA preparations using MALDI-quality standard peptides, prepared by successive dilution of a $1 \mu$ molar stock solution in $5 \%$ FA. The seven peptides in the mixture span a mass range of 1-7 $\mathrm{kDa}$ and originate from different peptides (see the Experimental section). Signals for all peptides were obtained in the range 100-50 fmol applied on the target with the matrix mixture and DHB preparations. The signals from the three peptides at $\mathrm{m} / \mathrm{z} 3159.4,5803.6$, and 6959.9 were weak or absent with the matrix mixture and DHB preparations at or below $25 \mathrm{fmol}$. With CHCA preparation, the signals for these three large peptides were absent in the full range studied. Similarly, the peptide at $\mathrm{m} / \mathrm{z} 1007.4$ was not observed with CHCA. It was of low intensity with the matrix mixture and $\mathrm{DHB}$ and not detectable below $50 \mathrm{fmol}$. Most likely ionization of this peptide is suppressed due to the absence of basic amino acids [38]. At the lowest level investigated, 6 fmol applied on the target, only two of the most intense peaks at $\mathrm{m} / \mathrm{z} 1084.4$ and 1281.7 were observed and the signal-to-noise ratio deteriorated dramatically. In general, the sensitivity and signal-to-noise ratio obtained with the matrix mixture and DHB preparations were comparable. However, the reproducibility upon successive acquisition of spectra is poor with DHB preparation.

Ribonuclease B was used for evaluating the sensitivity and performance of the matrix mixture preparation for analysis of proteins. Figure 9 shows MALDI/ linear TOF spectra obtained from a dilution series of $6 \mu$ molar stock solution in 5\% FA. SA preparation was included since it is considered to be the matrix of choice for proteins [39]. With $100 \mathrm{fmol}$ applied on the target, all spectra show the different glycosylated forms of ribonuclease B. Similar spectra were obtained at $50 \mathrm{fmol}$ with $\mathrm{DHB}$ and the matrix mixture preparations, while slight deterioration was observed with CHCA and SA preparations. At $25 \mathrm{fmol}$ applied on the target, the ion signal deteriorated dramatically with the matrix mix- 
(a) $\mathrm{CHCA}$

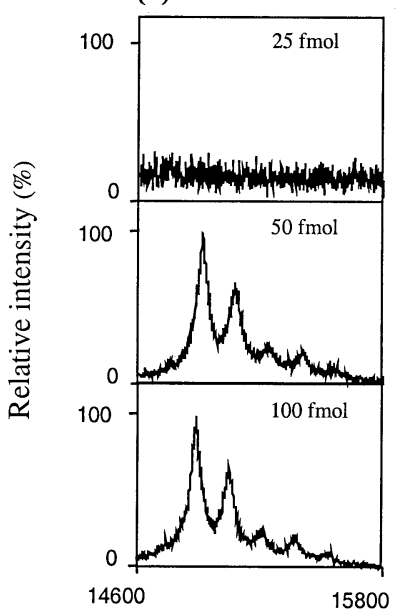

(b) DHB

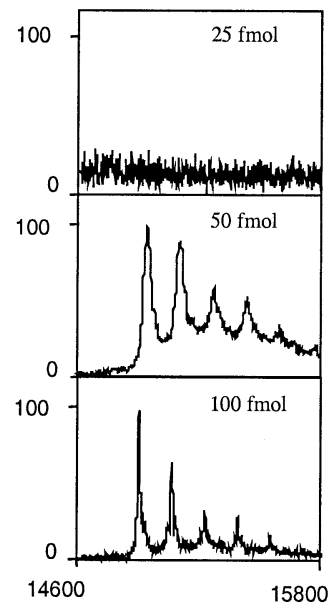

(c) SA

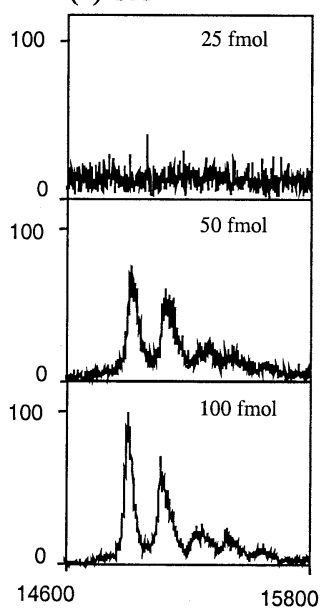

(d) Matrix mix

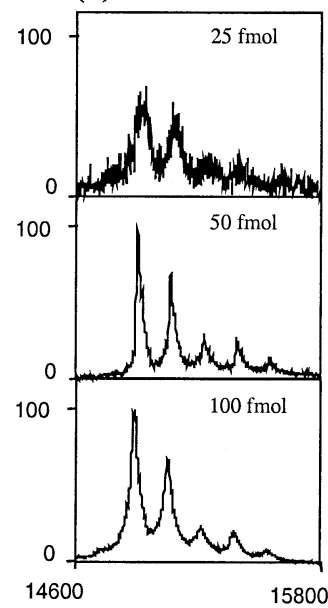

Figure 9. MALDI/Linear-TOF spectra obtained from a dilution series of ribonuclease B. $0.5 \mu \mathrm{L}$ of the protein was mixed with $0.5 \mu \mathrm{L}$ matrix solution, and applied onto the target in the dried-droplet method. The spectra were obtained with the preparations: (a) CHCA, (b) DHB, (c) SA, and (d) matrix mixture preparations. 100, 50 and 25 fmol were loaded on the target.

ture preparation and completely disappeared with CHCA, DHB, and SA preparations.

\section{Discussion}

In the present study we have investigated the use of a mixture of the two commonly used matrices, DHB and CHCA, for analysis of peptide mixtures as well as intact proteins. We have found that the matrix mixture results in slightly improved sequence coverage in peptide mass mapping in proteomics studies due to reduced chemical noise, compared to the use of either of the two matrices alone. In addition, the shot-to-shot reproducibility over the entire sample surface is dramatically improved, especially compared to the use of DHB matrix. This will result in a major advantage for automated data acquisition in high throughput proteomics studies. The use of the matrix mixture was also compared to concentration/desalting of in-gel digests on nano-columns followed by elution with matrix solution directly onto the MALDI target. In all cases studied, improved sequence coverage and comparable signal-to-noise levels were obtained using a simple dried-droplet preparation with the matrix mixture compared to inclusion of nanocolumn purification. The reason for the improved sequence coverage with the matrix mixture is that selective loss of small hydrophilic or large hydrophobic peptides, due to the use of the nano-column, is avoided. It is surprising that the use of the matrix mixture in dried-droplet preparation method results in a decrease of the chemical noise comparable to that obtained with nano-purification. It is our hypothesis that the biphasic crystallization results in a very homogeneous crystallization of CHCA in the central part of the sample spot, with small DHB crystals formed in the perimeter and exclusion of the salt contaminants to the outer rim of the prepared surface. This hypothesis is supported by the observation that CHCA type spectra are obtained from the central part and DHB type spectra from the perimeter. However, we have not been able to find any region in the entire preparation surface, which was dominated by salts and other contaminants. The fragmentation behavior supported the distribution of the crystals on the surface, thus the level of prompt and metastable decay comparable to that obtained with CHCA alone was observed in the central part and not in the perimeter. The amount of prompt fragmentation was even slightly more extensive for the matrix mixture than observed for preparations with only CHCA. The improved spot-to-spot reproducibility most likely is due to the formation of smaller crystals, homogeneously distributed over the surface, and a concomitant minimization of the variation in analyte-to-matrix ratio [29, 38].

The general sensitivity was found to be comparable for all the different preparation methods. However, for silver stained spots of very low intensity it was found advantageous to include a nano-column concentration step. The use of anchor chip targets [40] in combination with the matrix mixture preparation might result in a similar up-concentration of the analyte, but this was not tested in this study.

The matrix mixture preparation was also found advantageous for analysis of intact proteins. For glycoproteins, a pronounced improvement in signal intensity and resolution was observed, compared to the use of a single matrix component resulting in improved sensitivity and resolution of the glycan heterogeneity. The improvement observed for unmodified proteins was less pronounced. In addition, the matrix mixture preparation was found to tolerate considerable higher concentrations of urea in the sample solution. This might facilitate analysis of proteins with low solubility.

In conclusion, we have found that the matrix mixture 
offers several advantages for sample preparation. It is simple, fast, and spectra can be acquired at any position on the sample surface, simplifying fully automated data acquisition.

The advantages in proteomics studies might be considered marginal, when examined step-by-step in the procedure. Overall, these small improvements speed up sample preparation and data acquisition, and increase the sequence coverage resulting in improved fidelity in protein identification. The matrix mixture preparation showed surprising improvements in the quality of spectra for a number of intact proteins, most pronounced for glycoproteins, which were difficult to analyze with the previously used sample preparation methods.

The advantages of the matrix mixture preparation for proteomics studies and analysis of intact proteins have now been confirmed by a number of individuals in our and other laboratories using different mass spectrometers. Preliminary studies using MALDI MS/MS on either TOF-TOF or ion trap instruments indicate that the preparation method also facilitates sequencing studies. Mixing a "cold" and "hot" matrix (CHCA and 3-hydroxypicolinic acid) was also in a preliminary study found to allow analysis of cross-link products between peptides and oligonucleotides, which could not be observed with any of the single matrices.

\section{Acknowledgments}

The authors thank Andrea Lorentzen for technical assistance, Ole Østergaard (Carlsberg Laboratory, Copenhagen) for the barley 2-D gel, and Jane Noehr Larsen for the recombinant glucoamylase samples. They gratefully acknowledge Jonas Borch, Felix Elortza, Karin Hjernø, Sven Kjellstrom, and Thomas Jørgensen for helpful discussions. SL is supported by a Ph.D. fellowship under the SUE program from the Danish Research Councils. The work is part of the activities of the Center for Experimental Bioinformatics supported by the Danish National Research Foundation, and the Danish Biotechnology Instrument Center supported by the Danish Research Councils.

\section{References}

1. Hillenkamp, F.; Karas, M.; Beavis, R. C.; Chait, B. T. Anal. Chem. 1991, 63, 1193A-1202A.

2. Karas, M.; Bachmann, D.; Hillenkamp, F. Anal. Chem. 1985, 57, 2935-2939.

3. Karas, M.; Bachmann, D.; Bahr, U.; Hillenkamp, F. Int. J. Mass Spectrom. Ion Processes 1987, 78, 53-68.

4. Karas, M.; Hillenkamp, F. Anal. Chem. 1988, 60, 2299-2301.

5. Karas, M.; Ingendoh, A.; Bahr, U.; Hillenkamp, F. Biomed. Environ. Mass Spectrom. 1989, 18, 841-843.

6. Tanaka, K.; Waki, H.; Ido, Y.; Akita, S. Rapid Commun. Mass Spectrom. 1988, 2, 151-153.

7. Beavis, R. C.; Chait, B. T. Rapid Commun. Mass Spectrom. 1989, 3, 436-438.

8. Karas, M.; Bahr, U.; Ingendoh, A.; Nordhoff, E. Anal. Chem. Acta 1990, 241, 175-185.
9. Beavis, R. C.; Chait, B. T. Rapid Commun. Mass Spectrom. 1989, 3, 233-237.

10. Beavis, R. C.; Chait, B. T. Rapid Commun. Mass Spectrom. 1989, 3, 432-435.

11. Beavis, R. C.; Chait, B. T. Proc. Natl. Acad. Sci. U.S.A. 1990, 87, 6873-6877.

12. Strupat, K.; Karas, M.; Hillenkamp, F. Int. J. Mass Spectrom. Ion Processes 1991, 111, 89-102.

13. Beavis, R. C.; Chaudhary, T.; Chait, B. T. Org. Mass Spectrom. 1992, 27, 156-159.

14. Karas, M.; Bahr, U.; Strupat, K.; Hillenkamp, F. Anal. Chem. 1995, 67, 657-679.

15. Xiang, F.; Beavis, R. C. Rapid Commun. Mass Spectrom. 1994, 8 , 199-204.

16. Vorm, O.; Roepstorff, P.; Mann, M. Anal. Chem. 1994, 66, 3281-3287.

17. Yuqin, D.; Whittal, R. M.; Li, L. Anal. Chem. 1996, 68, $2494-$ 2500.

18. Gobom, J.; Nordhoff, E.; Mirgorodskaya, E.; Ekman, R. J. Mass Spectrom. 1999, 34, 105-116.

19. Onnerfjord, P.; Ekstrom, S.; Bergquist, J.; Nilsson, J. Rapid Commun. Mass Spectrom. 1999, 13, 315-322.

20. Zenobi, R.; Knochenmuss, R. Mass Spectrom. Rev. 1998, 17, 337-366.

21. Karas, M.; Gluckmann, M.; Schafer, J. J. Mass Spectrom. 2000, 35, 1-12.

22. Mormann, M.; Bashir, S.; Derrick, P. J.; Kuck, D. J. Am. Soc. Mass Spectrom. 2000, 11, 544-552.

23. Chou, J. Z.; Kreek, M. J.; Chait, B. T. J. Am. Soc. Mass Spectrom. 1994, 5, 10-16.

24. Xu, N.; Huang, Z. H.; Watson, T.; Gage, D. A. J. Am. Soc. Mass Spectrom. 1997, 8, 116-124.

25. Yao, J.; Scott, J. R.; Young, M. R.; Wilkins, C. H. J. Am. Soc. Mass Spectrom. 1998, 9, 805-813.

26. Amini, A.; Dormady, S. J.; Riggs, L.; Regnier, F. E. J. Chromatogr. A 2000, 894, 345-455.

27. Bornsen, K. O. Methods in Molecular Biology. Vol. CXLVI, Protein and Peptide Analysis: New Mass Spectrometric Applications; In: Chapman, J. R., Ed.; Humana Press: Totowa, NJ, 2000; p. 387.

28. Billeci, T. M.; Stults, J. T. Anal. Chem. 1993, 65, 1709-1716.

29. Gusev, A. I.; Wilkinson, W. R.; Proctor, A.; Hercules, D. M. Anal. Chem. 1995, 67, 1034-1041.

30. Rosinke, B.; Strupat, K.; Hillenkamp, F.; Rosenbusch, J. J. Mass Spectrom. 1995, 30, 1462-1468.

31. Pfenninger, A.; Karas, M.; Finke, B.; Stahl, B. J. Mass Spectrom. 1999, 34, 98-104.

32. Fratelli, M.; Demol, H.; Puype, M.; Casagrande, S.; Eberini, I.; Salmona, M. PNAS 2002, 99, 3505-3510.

33. Shevchenko, A.; Wilm, M.; Vorm, O.; Mann, M. Anal. Chem. 1996, 68, 850-858.

34. Beavis, R. C.; Chait, B. T. Anal. Chem. 1990, 62, 1836-1840.

35. Jensen, O. N.; Larsen, M. R.; Roepstorff, P. Proteins: Structure, Function, and Genetics 1998, (Suppl. 2), 74-89.

36. Cohen, S. L.; Chait, B. T. Anal. Chem. 1996, 68, 31-37.

37. Reid, G. E.; Stephenson, J. L.; McLuckey, S. A. Anal. Chem. 2002, 74, 577-583.

38. Amado, F. M.; Domingues, P.; Santana-Marques, M. G.; Ferrer-Correia, A. J. Rapid Commun. Mass Spectrom. 1997, 11, 1347-1352.

39. Kussmann, M.; Nordhoff, E.; Rahbek-Nielsen, H.; Haebel, S. J. Mass Spectrom. 1997, 32, 1-9.

40. Gobom, J.; Schuerenberg, M.; Muller, M.; Theiss, D.; Lehrach, H.; Nordhoff, E. Anal. Chem. 2001, 73, 434-438. 